\title{
Differential Expression of Toll-like Receptor 4 in Healthy and Diseased Human Gingiva
}

\author{
J.-P. $\mathrm{Li}^{1,2}$, Y. Chen ${ }^{1}$, C. H. C. $\mathrm{Ng}^{1}$, M.-L. Fung ${ }^{3}$, A. $\mathrm{Xu}^{4}$, B. Cheng ${ }^{2}$, S. W. Tsao ${ }^{5}$, W. K. \\ Leung $^{1}$ \\ ${ }^{1}$ Faculty of Dentistry, The University of Hong Kong, Hong Kong SAR, China; ${ }^{2}$ Guanghua \\ School of Stomatology, Sun Yat-Sen University, Guangzhou, China; ${ }^{3}$ Department of \\ Physiology, ${ }^{4}$ Department of Medicine and ${ }^{5}$ Department of Anatomy, Li Ka Shing Faculty of \\ Medicine, The University of Hong Kong, Hong Kong SAR, China.
}

Correspondence: W. Keung Leung, Room 1B25, Prince Philip Dental Hospital, Faculty of Dentistry, The University of Hong Kong, 34 Hospital Road, Hong Kong SAR, China.

Tel: +852-2859-0417

Fax: $+852-2858-2532$

e-mail: ewkleung@hkucc.hku.hk.

Running head: TLR4 in human gingiva

The authors report no conflict of interest related to this study.

This project was supported by the University of Hong Kong Small Project Funding (200807176129, 201007176307) and Seed Funding (200911159126). 


\title{
Word counts
}

\begin{abstract}
: 250;
Body (excluding abstract, references, and figure legends): 4494.
\end{abstract}

Figures: $5+1$ supplementary figure

Number of references: 23

Date of resubmission: January 7, 2014 
Li J-P, Chen Y, Ng CHC, Fung M-L, Xu A, Cheng B, Tsao SW, Leung WK. Differential expression of Toll-like receptor 4 in healthy and diseased human gingiva. J Periodont Res

\begin{abstract}
Background and Objective: Lipopolysaccharide (LPS)-mediated signaling in host cells involves Toll-like receptor 4 (TLR4), accessory molecules including LPS-binding protein (LBP), CD14 and MD-2. However, expression of these innate defense molecules in various compartments of human periodontium remained unclear. This study aims to investigate the expression profile of TLR4 in human gingiva.
\end{abstract}

Material and Methods: Human gingival biopsies were collected from healthy gingival or chronic periodontitis tissue. Primary gingival keratinocytes and fibroblasts were cultured. Immunohistochemical analysis for TLR4 was performed. Transcripts of TLR4, MD-2, CD14 and LBP, and their protein products were examined using reverse transcription PCR, immunoprecipitation and immunoblotting. The interactions between these molecules in keratinocytes and fibroblasts were investigated by co-immunoprecipitation.

Results: TLR4 immunoreactivity was found in healthy gingival epithelium and periodontitis tissue, which appeared lower in junctional epithelium $(P \leq 0.01)$. Fibroblasts and inflammatory cells stained more intensively for TLR4 in diseased periodontal tissues $(P<0.001)$. Three TLR4 splicing variants, two MD-2 splicing variants, and one CD14 mRNA were expressed 
by gingival keratinocytes and fibroblasts. Protein expression of TLR4, CD14 and MD-2 were detected in keratinocytes and fibroblasts in vitro. TLR4 protein from gingival keratinocytes and fibroblasts could be co-immunoprecipitated with CD14 or MD-2, suggesting the association of the related molecules in vivo. LBP transcript was detected in gingival biopsies but not in primary cultures of gingival keratinocytes or fibroblasts.

Conclusion: TLR4, CD14 and MD-2, but not LBP, were expressed in human gingival keratinocytes and fibroblasts. TLR4 expression level in junctional epithelium appeared to be the lowest within periodontal epithelial barrier.

Key words: Antigens, CD14; chronic periodontitis; fibroblasts; keratinocytes; lymphocyte antigen 96; Toll-like receptor 4 
Lipopolysaccharides (LPS) as cell wall components of gram-negative periodontopathogenic bacteria, are conserved structural motifs known as pathogen associated molecular pattern (PAMP). They are important factors for mediating the host immune response that initiates inflammatory changes during periodontitis. The pathological effects of LPS is mainly mediated through molecules expressed on host cells such as Toll-like receptor 4 (TLR4) alongside co-receptor cluster of differentiation 14 (CD14), accessory molecule lymphocyte antigen 96 (MD-2) in the presence of a liver derived soluble acute-phase protein or the LPS-binding protein (LBP). The recognition of LPS by the host cells is believed to be first initiated by LBP which specifically binds LPS. The LPS bound LBP interacts with membrane bound CD14 on the target cell to form a complex, which the latter was transferred to the MD-2 accessory molecule forming a trimeric LPS/MD-2/TLR4 complex. The MD-2 accessory molecule is a secretary glycoprotein associated with the ectodomain of TLR4. The LPS receptor-complex initiates the recruitment of adapter proteins to the intracellular domains of TLR4, which in turn triggers the signaling cascade leading to the nuclear translocation of transcription factors and the production of inflammatory cytokines (1). In this system, TLR4 and MD-2 are essential for the LPS recognition and function. Previous report demonstrated that Aggregatibacter actinomycetemcomitans, Fusobacterium nucleatum, Porphyromonas gingivalis, Prevotella intermedia, Prevotella nigrescens, Tannerella forsythensis and Veillonella parvula stimulated human embryonic kidney cells through CD14-TLR2, but only A. actinomycetemcomitans and V. parvula stimulated the same cells through CD14-TLR4 (2).

Many studies addressed the expression of LBP, TLR4, CD14 and MD-2 in human 
gingival tissues, but these findings appeared inconsistent (3-10). While some studies reported TLR4 in immune cells of gingival connective tissues $(8,10)$, others presented with immunohistochemical evidence that TLR4 was expressed in human gingival epithelium $(3,7$, 9), especially in the basal cell layer (9); and the expression was enhanced in periodontitis $(3,7$, 9, 10). These studies, however, did not describe any distinctive TLR4 expression profiles of junctional epithelium, oral sulcular epithelium or oral epithelium. On the other hand, both human TLR4 $(11)$ and MD-2 $(12,13)$ genes were reported to encode several alternatively splicing transcript variants, however their corresponding expression from residential cells isolated from gingival tissue has not yet been reported. In the present study, we defined different in vivo expression profiles of TLR4 in healthy and periodontitis-involved human gingiva followed by examination of the transcript variants and protein products of TLR4, CD14 and MD-2 in cultured periodontitis-free primary human gingival epithelial cells and fibroblasts.

The hypothesis of this study is that TLR4 as one pattern recognition receptor against LPS is expressed and detectable as innate defense element in all compartments of the human periodontium.

\section{Material and methods}

\section{Periodontal biopsies}

The study was approved by the Institutional Review Board of The University of Hong Kong/Hospital Authority Hong Kong West Cluster. The gingival tissue sampling procedure 
has been reported in our previous study $(14,15)$. Non-smoking Chinese patients between 35 and 60 years old, who attended Prince Philip Dental Hospital, the University of Hong Kong, were recruited to this study between September 2006 and January 2009. Participants had to be systemically healthy, did not have prior periodontal treatment, and had not received antibiotics or anti-inflammatory drugs in the previous 6 months. The purpose of the study and the procedures to be involved were explained to the participants and informed consent was obtained.

The inclusion criteria for the periodontitis group were as follows: 1) at least two teeth per quadrant showed untreated chronic periodontitis with probing pocket depth (PPD) $>6$ $\mathrm{mm}$ and probing attachment level $(\mathrm{PAL})>5.0 \mathrm{~mm} ; 2)$ radiographic evidence of alveolar bone loss; and 3) at least one periodontally involved tooth was scheduled for extraction or periodontal surgery. The inclusion criteria for the control group were as follows: 1) no sites with $\mathrm{PPD} \geq 3 \mathrm{~mm}$ or $\mathrm{PAL} \geq 1 \mathrm{~mm} ; 2$ ) no radiographic evidence of periodontal bone loss $>$ $10 \%$ or any furcation involvement in multi-rooted teeth; 3 ) full-mouth bleeding on probing score $\leq 30 \%$, and (4) a tooth scheduled for crown-lengthening surgery or extraction for orthodontic or prosthodontic reasons. To obtain gingival biopsies with intact junctional or pocket epithelium, gingival biopsies were collected through internal beveled incision extended below marginal periodontal bone into periodontal space as deep as possible, as described in our previous report (15).

Forty-four gingival biopsies were obtained from patients with advanced chronic periodontitis $(46.3 \pm 9.1$ years; 23 females $)$. Immunohistochemistry, immunoprecipitation and 
immunoblotting were performed on 38, 3 and 3 samples, respectively. Thirty-four control samples were obtained from healthy subjects $(42.2 \pm 5.8$ years; 19 females $)$. Immunohistochemistry, reverse transcription polymerase chain reaction (RT-PCR), immunoprecipitation and immunoblotting were performed on 10, 5, 3 and 3 samples, respectively. Cell explants (isolation of both human gingival fibroblasts and keratinocytes) were prepared from thirteen control samples. Out of them, RT-PCR, immunoprecipitation and immunoblotting were performed on cells from 9, 7 and 10 samples, respectively.

\section{Immunohistochemistry}

Samples were fixed in $10 \%$ neutral buffered formalin solution for 24 hours before being dehydrated and embedded in paraffin blocks. Sections ( $4 \mu \mathrm{m}$ thickness $)$ were cut and mounted on silicon-coated slides. After the sections were dewaxed and rehydrated, they were treated with $3 \%$ hydrogen peroxide and heated in a buffer $(0.05 \%$ Tween $20,1 \mathrm{mM}$ EDTA, $10 \mathrm{mM}$ Tris $\mathrm{HCl}, \mathrm{pH} 9.0$ ) at $98^{\circ} \mathrm{C}$. After blocking in $2.5 \%$ horse serum for 20 minutes, sections were incubated with primary antibodies against TLR4 (Rabbit anti-human polyclonal IgG, 1:100, sc-10741, Santa Cruz Biotechnology Inc., Santa Cruz, CA.) in $1 \%$ bovine albumin at $4^{\circ} \mathrm{C}$ overnight, then stained (ImmPRESS ${ }^{\mathrm{TM}}$ Universal Reagent, MP-7500, Vector Laboratories, Burlingame, CA) and visualized using a diaminobenzidine (DAB) solution. Sections incubated with control normal rabbit IgG (1:100) instead of the primary antibodies were also processed at the same time, which were all stained uniformly negative.

Stained sections were analyzed with a digital imaging system (Leica DC 300 Ver 2.0, 
Leica, Wetzlar, Germany) and Leica Qwin Standard V 2.6 software (Leica, Wetzlar, Germany). For biopsies of healthy group $(n=10)$, gingival epithelium was divided into three compartments: junctional epithelium (H-JE), oral sulcular epithelium (H-OSE), and oral epithelium (H-OE). Similarly, gingival epithelium of periodontitis group $(n=38)$ was also divided into two compartments: pocket epithelium (P-PE) and oral epithelium (P-OE), according to their spatial position relative to the free gingival margin. The TLR4 expression in different epithelium compartments was evaluated and quantitatively analyzed as the proportion of positively stained area over the total area of the defined epithelium compartment and presented as area (\%) (supplementary Fig. S1). The proportion of subepithelial cells which were TLR4-immunoreactive was calculated and presented as count (\%) according to a protocol described in a previous study (14).

\section{Cell culture}

To obtain pure primary human gingival keratinocytes without contamination from fibroblasts, the healthy gingival tissue (HGT) biopsies $(n=13)$ were washed in ice-cooled phosphate-buffered saline (PBS) and incubated in $2 \mathrm{U} / \mathrm{ml}$ dispase (Cat. No. 17105, GIBCO ${ }^{\mathrm{TM}}$ Invitrogen, Carlsbad, CA) diluted in serum-free Dulbecco's Modified Eagle Medium, (DMEM, Cat. 12800-017, GIBCO ${ }^{\mathrm{TM}}$ Invitrogen, Carlsbad, CA) at $4^{\circ} \mathrm{C}$ overnight. The epithelium was then mechanically separated from the subepithelial layer as an intact sheet using forceps. The epithelium sheet was minced and seeded in a 6-cm diameter culture dish and covered with a sterile $22 \times 22 \mathrm{~mm}$ glass coverslip. The tissue blocks were initially cultured in Oral 
Keratinocyte Medium (OKM, Cat. 2611, ScienCel1 ${ }^{\mathrm{TM}}$ Research Laboratories, Carlsbad, CA) supplemented with $10 \%$ fetal bovine serum (FBS, Cat. 10437-036, GIBCO ${ }^{\mathrm{TM}}$ Invitrogen, Carlsbad, CA). When cells started to grow out of the tissue blocks, FBS was immediately removed from the culture medium and the cells and tissue blocks were maintained in OKM. Cells at $80 \%$ confluence were passaged into a $10-\mathrm{cm}$ diameter culture dish. The cultures were then purged of any fibroblast contamination by treating with $0.25 \%$ Trypsin in PBS for 3 minutes. The detached cells were re-seeded in a 6-well culture plate. The purification procedure was repeated every other day until no more spindle-shaped cells were found to grow in the 6-well culture plate for two successive treatments. Human primary gingival fibroblasts were cultured from the de-epithelialized gingival tissues, which were minced and seeded in DMEM containing 10\% FBS. Both human gingival keratinocytes (HGK) and fibroblasts (HGF) from the third passage were used in the following experiments.

\section{Reverse transcription Polymerase Chain Reaction}

Total RNA was extracted from cultured cells ( $\mathrm{n}=9$ independent cell explants) and periodonotitis-free gingival biopsies $(n=5)\left(\right.$ RNeasy $^{\circledR}$ Mini Kit, Qiagen, Valencia, CA.). First-strand complementary DNA (cDNA) was then synthesized (Superscript First Chain Synthesis Kit, Invitrogen, Carlsbad, CA). To amplify the target cDNAs using polymerase chain reaction (PCR), oligonucleotide primer pairs were designed with PerlPrimer v1.1.17 (http://perlprimer.sourceforge.net). The RefSeq names of the Target transcripts, their accession numbers in GenBank and the sequences of the oligonucleotide primer pairs were listed below: 
LBP (NM_004139; Forward: 5'-TCT AAT ATC CGA CTG ACC AC-3', Reverse: 5'-CAG

CTC TGC ATT GAA TAG TC-3'), CD14 (NM_000591; Forward: 5'-ACT TAT CGA CCA TGG AGC G-3', Reverse: 5'-AAG CTG GAA AGT GCA AGT C-3'), MD-2 (NM_015364 for MD-2.1; NM_001195797 for MD-2.2; Forward: 5'-CTC AGA AGC AGT ATT GGG T-3', Reverse: 5'-AAT TAG GTT GGT GTA GGA TGA C-3'), TLR4 (NM_138554 for TLR4.1; NM_003266 for TLR4.3; NM_138557 for TLR4.4; Forward: 5'- GTC AGA CGG TGA TAG CGA G-3', Reverse: 5'-GAT GTC CAA TGG GGA AGT T-3'), and GAPDH (NM_002046; Forward: 5'-CAA CTT TGG TAT CGT GGA AGG A-3', Reverse: 5'-AAG GTG GAG GAG TGG GTG TCG-3'). The cycling conditions were $95^{\circ} \mathrm{C}$ for 30 seconds, $58^{\circ} \mathrm{C}$ for 30 seconds and $72^{\circ} \mathrm{C}$ for 45 seconds for 37 cycles. All amplicons were gel purified and sequenced to confirm their identity.

\section{Immunoprecipitation and co-immunoprecipitation}

Cells $\left(n=1.0 \times 10^{7}\right.$ from seven culture explants) and gingival biopsies $(n=3$ each for periodontitis and healthy tissue) were lysed with lysis buffer (1\% Triton-X100, $150 \mathrm{mM} \mathrm{NaCl}$, $20 \mathrm{mM}$ Tris $\mathrm{HCl}, \mathrm{pH} 7.4$, and $2 \mu \mathrm{L} / \mathrm{mL}$ protease inhibitor, P8340, Sigma-Aldrich, St. Louis, $\mathrm{MO}$ ), and homogenized on ice. After centrifugation at $12,000 \times g$ for 20 minutes at $4^{\circ} \mathrm{C}$, the supernatant was collected and protein concentrations determined (BCA kit, Pierce Biotechnology, Hercules, CA). For immunoprecipitation and co-immunoprecipitation, $500 \mu \mathrm{g}$ of total cellular protein, or $800 \mu \mathrm{g}$ of total tissue protein or $500 \mu \mathrm{g}$ BSA in $500 \mu \mathrm{L}$ protein extraction buffer (negative control) were used. 
As a positive control, human peripheral blood mononuclear cells (PBMC) were isolated using Ficoll-Paque ${ }^{\mathrm{TM}}$ Premium (Cat. No. 17-5442-02, GE Healthcare Bio-Sciences AB, Uppsala, Sweden). Briefly, fresh blood collected from a volunteer was immediately supplemented with EDTA to a final concentration of $1.5 \mathrm{mg} / \mathrm{mL}$, and then diluted with equal volume of PBS. Four milliliters of the diluted blood sample were carefully layered on $3 \mathrm{~mL}$ of Ficoll-Paque ${ }^{\mathrm{TM}}$ Premium solution in a centrifuge tube. After centrifugation at $400 \times g$ for 30 minutes at $18^{\circ} \mathrm{C}$, the $\mathrm{PBMC}$ layer between the upper plasma/platelets and the lower Ficoll-Paque ${ }^{\mathrm{TM}}$ Premium layers was collected and suspended in $6 \mathrm{~mL}$ of PBS. After 3 cycles of washing and centrifugation in PBS, the PBMC was pelleted and lysed according to the aforementioned protocol. Four hundred micrograms of the crude PBMC lysate were used in immunoprecipitation and co-immunoprecipitation as positive controls.

All protein specimens were pre-cleared by addition of $1.0 \mu \mathrm{g}$ of an appropriate control IgG (mouse, rabbit or goat) corresponding to the host species of the primary antibody, together with $20 \mu \mathrm{L}$ of re-suspended Protein A/G PLUS-Agarose beads (sc-2003, Santa Cruz Biotechnology Inc., Santa Cruz, CA). After incubation at $4^{\circ} \mathrm{C}$ for 30 minutes, samples were centrifuged at $2,500 \times g$ for 2 minutes. The supernatant was collected and $1 \mu \mathrm{g}$ of antibody against either CD14 (sc-1182, Santa Cruz Biotechnology Inc., Santa Cruz, CA) or MD-2 (sc-20668, Santa Cruz Biotechnology Inc., Santa Cruz, CA) or TLR4 (goat anti-human polyclonal antibody, ab53629, ABcam, Cambridge, MA) was added together with $20 \mu \mathrm{L}$ of resuspended Protein A/G PLUS-Agarose beads. The samples were incubated at $4^{\circ} \mathrm{C}$ overnight. After three washes, immunoprecipitates were eluted in $1 \times$ electrophoresis sample buffer and 
run on sodium dodecyl sulfate-polyacrylamide gel electrophoresis (SDS-PAGE), then analyzed by immunoblotting.

\section{SDS-PAGE and immunoblotting}

For SDS-PAGE, CD14 [500 $\mu \mathrm{g}$ of concentrated (Centrifugal Filter Devices YM-50, Millipore, Bedford, MA) total protein], MD-2 (20 $\mu \mathrm{g}$ of total protein) and TLR4 (protein immunoprecipitates) samples were separated by $12 \%$ (for CD14 and MD-2) or $7.5 \%$ (for TLR4) SDS-PAGE before being transferred onto polyvinylidene difluoride membranes. After blocking in a buffer $(0.05 \%$ Tween $20,5 \%$ skimmed milk, $150 \mathrm{mM} \mathrm{NaCl}, 50 \mathrm{mM}$ Tris $\mathrm{HCl}$, $\mathrm{pH}$ 7.2), the membranes were incubated overnight with antibodies against CD14 (1:1000) or MD-2 (1:500, sc-20668, Santa Cruz Biotechnology Inc., Santa Cruz, CA) or TLR4 (1:1000, Cat. MAB1478, R\&D, Minneapolis, MN). The blots were visualized using a detection kit (WesternBreeze ${ }^{\circledR}$ Chromogenic Immunodetection Kit, Invitrogen, Carlsbad, CA).

\section{Statistical analyses}

Data were analyzed using a statistical package (IBM SPSS 20, Armonk, New York, USA). As the normality (checked by Kolmogorow-Smirnoff test) of the data appeared to be valid, one-way ANOVA was used to examine the difference between six groups on area $(\%)$ of TLR4 expression. Post hoc multiple comparisons were performed using Bonferroni tests. For the proportion of subepithelial cells, $t$-test was used to examine differences between the control and test groups. The alpha level was set at 0.05 . 


\section{Results}

\section{Localization of TLR4 protein in human gingiva}

In both healthy (Fig. 1) and periodontitis-involved (Fig. 2) periodontal soft tissue, TLR4-immunoreactivity could be readily observable in oral epithelium (Fig. 1b, Fig. 2b \& 2d), oral sulcular epithelium (Fig. 1b, 1c \& 1e), pocket epithelium (Fig. 2a, 2e \& 2g), while it appeared lesser expressed in junctional epithelium (Fig. 1d). The TLR4-immunoreactivity appeared to be isolated to the cell membrane (Fig. 1e \& Fig. 2d). Statistical analysis demonstrated difference in the mean area (\%) of TLR4 expression among H-JE, H-OE, H-OSE, P-OE and P-PE epithelial compartments (Fig. 3) while post hoc multiple comparisons confirmed that TLR4 area (\%) were similar among H-OE $(34.5 \pm 8.2 \%), \mathrm{H}-\mathrm{OSE}$ $(30.3 \pm 8.8 \%)$ and P-OE $(31.5 \pm 8.7 \%)$, but were significantly higher than H-JE $(16.2 \pm 7.6 \%)$ $(P \leq 0.01$, Bonferroni multiple comparisons; Fig. 3). The TLR4 area (\%) expression in P-PE $(25.2 \pm 12.5 \%)$ did not appear to be statistically different than that of any other epithelial compartment in health or disease (Fig. 3).

In the subepithelial regions, fibroblast-like cells in healthy gingiva were mostly unstained for TLR4 (Fig. 1c \& 1d), whereas many fibroblast-like cells in periodontitis tissue were heavily stained for TLR4, particularly those proximal to the inflammatory infiltration (Fig. 2f \& 2i).

In healthy gingiva, leukocyte-like cells were sparsely scattered in oral sulcular 
epithelium, junctional epithelium, and the subepithelial regions (Fig. 1c \& 1d). These cells were mostly negative for TLR4, except those with dendritic morphology. In periodontitis tissue, epithelium-infiltrating leukocyte-like cells were largely negative for TLR4, but those accumulating in the subepithelial connective tissue were intensely stained (Fig. $2 g$ \& 2i). Some TLR4-positive leukocyte-like cells appeared to accumulate around a mass of TLR4-negative inflammatory cells (Fig. 2h \& 2k). The TLR4-immunoreactivity in endothelial cells was very weak or absent (Fig. $1 \& 2$ ).

The proportion of subepithelial cells that were TLR4-immunoreactive was significantly higher in periodontitis tissue than in healthy gingiva $(68.4 \pm 13.7 \%$ in 38 periodontal pockets vs. $12.3 \pm 5.6 \%$ in 10 control samples; $P<0.001$, $t$-test).

\section{Transcript expression of TLR4, MD-2, CD14 and LBP in human gingival keratinocytes, fibroblasts and tissues}

The human TLR4 gene contains two alternative splicing exons (Fig. 4a), each of which may be present or absent in the final mature transcript, theoretically resulting in four mRNA splicing variants (Fig. 4b). We identified the splicing variants by RT-PCR using pairs of primers designed to amplify the splicing regions containing the exons. Using this method, three splicing variants, TLR4.1 (amplicon size: 518bp), TLR4.3 (amplicon size: 638bp) and TLR4.4 (amplicon size: 351bp) were identified in HGK, HGF and HGT (Fig. 4c). However, TLR4.2 splicing variant (amplicon size: 474bp) was not found despite repeated experiments.

Similarly, MD-2 gene has one alternative splicing exon giving rise to two MD-2 splicing 
variants. Both $M D-2.1$ (amplicon size: 422bp) and $M D-2.2$ (amplicon size: 333bp) were detected in HGK, HGF and HGT, but $M D-2.1$ appeared to be more prominent (Fig. 4d). Identity of the PCR products of these TLR4 and MD-2 splicing variants were further confirmed by gel-purification and subsequent DNA-sequencing (data not shown).

CD14 mRNA was detected in HGK, HGF and HGT (Fig. 4e). LBP mRNA could only be detected in HGT but not in HGK or HGF (Fig. 4f).

\section{Protein expression of TLR4, CD14 and MD-2 in human gingival tissue and cells and} their association in HGF and HGK

The TLR4 protein was detected by immunoprecipitation and immunoblotting from the lysate of HGF, HGK, HGT and periodontitis gingival tissue (PGT) and was found to have a molecular mass of about $120 \mathrm{kDa}$ (Fig. 5c). The CD14 and MD-2 proteins were detected in HGF, HGK, HGT and PGT by immunoblotting showing molecular mass of $55 \mathrm{kDa}$ and 20 kDa, respectively (Fig. 5a \& 5b). The authors did not attempt to quantity CD14, MD-2 and TLR4 peptide expression from HGF or HGK cultures. However, the research group did not observe varied detectability of CD14, MD-2 and TLR4 from HGF of the ten control specimens, or the three HGT/PGT tissue biopsies.

In human gingival fibroblasts and keratinocytes, both CD14 and MD-2 could be individually co-immunoprecipitated with TLR4 protein (Fig. 5B), suggesting associations of TLR4/CD14 and TLR4/MD-2. However, CD14 and MD-2 did not co-immunoprecipitate, indicating that these two HGF and HGK molecules did not form a direct or indirect complex 
with each other in vitro (data not shown).

\section{Discussion}

The innate extracellular signaling pathway of LPS pattern recognition mainly involves the interaction of co-receptor CD14, accessory molecules LBP, MD-2 and corresponding PAMP receptor TLR4. Many studies attempted to investigate the expression of these proteins in human gingival cells and tissues - the results have been to some extent confusing. For example, although the in vitro expression of TLR4 in gingival keratinocytes and fibroblasts has been reported (3-6), previous immunohistochemical studies yielded inconsistent results regarding the in vivo gingival distribution of $\operatorname{TLR} 4(3,7-10)$. While in some studies $(8,10)$ TLR4 was reported in immune cells of gingival connective tissues, others $(3,7,9)$ presented with evidence that TLR4 could be identified in human gingival epithelium and enhanced in periodontitis. None of these studies described any distinctive TLR4 expression profiles on junctional epithelium, oral sulcular epithelium and oral epithelium, which play distinctive roles in the pathogenesis of periodontitis. In the present study, we defined in details the expression profiles of TLR4 in healthy and periodontitis-involved human gingiva and in junctional, oral sulcular, oral and pocket epithelium, followed by examination of the transcript and protein products of TLR4, CD14 and MD-2 in primary gingival cell cultures from healthy subjects, and periodontal soft tissues biopsies from health and disease.

Some previous studies reported that periodontitis associates with increased expression of 
TLR4 in leukocytes (8) and epithelial cells (5) in human gingiva. However, in the present study, although apparently we confirmed that a higher proportion of leukocyte-like cells were stained positive for TLR4 in periodontitis than in healthy control (Fig. $1 \& 2$ ), the difference in epithelial expression of TLR4 between corresponding healthy or periodontitis compartments does not appear statistically significant (Fig. 3). The lack of difference between H-JE and P-PE, despite the latter appeared to express higher relative TLR4 level is probably a result of the limited sample size of this study. Nevertheless, for the first time, we described comprehensively the differential expressions of TLR4 protein in the periodontal epithelial barrier. Intriguingly, TLR4 expression appeared to be lower in junctional epithelium, compared to oral epithelium and oral sulcular epithelium (Fig. 3). It should be noted that we did not try to confirm these results in the subsequent in vitro experiments because currently these are no reliable methods to induce the cultured gingival epithelial cells to differentiate specifically toward junctional, oral sulcular or oral epithelial morphotypes. Nevertheless, how this distinctive expression profile of TLR4 in human gingival epithelium impacts on periodontal pathogenesis warrants further studies. We believe these results suggest the possibility that at least in healthy gingiva different epithelial compartments may be programmed to sense LPS challenge differently.

Possibly due to the lower expression level of TLR4 peptide in gingiva, the effort of identifying cell types in periodontal tissues using double immunofluorescence methods failed (data not shown). As a consequence, cell-type identification is mainly based on cell morphology and location in the tissue section where certain cells were observed. The 
expression of TLR4 in some leukocyte subpopulations was preliminarily demonstrated in periodontitis tissues (Fig. 2c \& 2d). The epithelium-infiltrating cells in both healthy and periodontitis tissues (possibly neutrophils and $\gamma \delta$-T cells according to the cell localization) showed little staining for TLR4 (Fig. 1c, 1d, 2c \& 2i). Specifically, the immune cells present in the healthy gingiva were mostly negative for TLR4 (Fig. 1c \& 1d). Further studies to profile the transcript/peptide expression of TLR4, CD14 and MD-2 in HGT in vivo, in particular those from residential and defense cells may help shed light on the biological relevance of all cells capable of PAMP recognition in human periodontium.

Human TLR4 gene contains two alternative exons (exon $a$ and $b$ ) that can be present or absent in the final mature mRNA (Fig. 4a), theoretically forming four splicing variants. These variants are products of two splicing exons inserted together, inserted individually or absent in the open reading frame (ORF) of TLR4 at $93 \mathrm{bp}$ downstream of the translation starting site (Fig. $4 \mathrm{~b})$. Exon $b$ is required to maintain the integrity of ORF, but loss of exon $b$ or presence of exon $a$ results in frame-shifting or premature in-frame stop codon, respectively. Only TLR4.1 that contains exon $b$ can be translated into the full-length functional protein. Initially, all the four TLR4 splicing variants were reported observable from human monocytes (11). But now it is believed that existence of variant TLR4.2 (GenBank accession number: NM_138556) is insufficiently supported. As a result, NM_138556 has been permanently removed from GenBank database (16).

Using RT-PCR, we identified the expression of TLR4.1, TLR4.3 and TLR4.4 transcript variants in healthy human gingival tissues and cultured gingival keratinocytes and fibroblasts. 
Currently, there is no data regarding the precise biological significances of these splicing variants expression in human. However, a similar alternatively spliced mouse TLR4 mRNA was reported to produce a truncated protein, which competitively inhibits TLR4 signaling (17). Our findings show these three variants are present in gingival cells and tissues at comparable levels (Fig. 4c). Therefore, when evaluating the transcriptional level of the TLR4 gene, the non-protein coding transcripts of TLR4.3 and TLR4.4 should be detected and quantified to take into account and avoid any possible bias or misinterpretation.

For the first time, we have identified the TLR4 glycoprotein in both human gingival keratinocytes and fibroblasts using immunoprecipitation and immunoblotting. All immune-detected human glycopeptides molecular sizes were in agreement with reports in the literature (please see below). Furthermore, the in vitro expressions of signals for the above glycoproteins were characterized using the same HGF explant. The later substantiated in part the validity of our glycoprotein interaction experiments. We also considered periodontitis tissue could function as positive control because of its heavy inflammatory cells infiltration meaning proteins like CD14, MD-2 and TLR4 expressed by macrophages, monocytes and other leukocytes could be readily isolated or detected $(8,10)$. Negative control for HGF explant study carried out by replacing protein samples with BSA in protein extraction buffer.

Probably dependent on the amount of glycosylation, the molecular mass of the TLR4 could be 96 or $120 \mathrm{kDa}$. Glycosylation is reported to be closely involved with the biological function of the TLR4 receptor complex by affecting its cell surface localization, protein complex formation, and LPS responsiveness (18). In this study, human gingival tissue as well 
as cultured keratinocytes and fibroblasts were found to express the $120 \mathrm{kDa}$ TLR4 protein, which has been reported to be the most biologically active form among the different glycosylated variants (18).

Furthermore, the TLR4 protein in gingival fibroblasts and keratinocytes co-immunoprecipitated with MD-2 or CD14 from the same cellular source (Fig. 5e-g), confirming the in vitro presence of TLR4/CD14 and TLR4/MD-2 complexes, as hypothesized in previous reports (19-21). However, CD14 and MD-2 did not co-immunoprecipitate and did not form complexes in vitro (data not shown) suggesting that these two HGF or HGK originating molecules are not directly associated with each other (1).

Two MD-2 splicing variants were identified in gingival cells and tissue (Fig. 4d). The shorter $M D-2.2$ variant lacks the splicing exon compared to the longer $M D-2.1$ variant (12). It is believed that $M D-2.2$ negatively regulates TLR4-signaling by competitively inhibiting the binding of $M D-2.1$ protein to TLR4 (22). However, in this study only a single band of $M D-2.1$ protein of around $20 \mathrm{kDa}$ was detected (Fig. 5b). Possibly the mRNA levels of $M D-2.2$ were much lower than that of $M D-2.1$ (Fig. 4d) resulting in an undetectable $M D-2.2$ protein expression level. It has been reported that inflammatory cytokines such as IFN- $\gamma$ and IL-6 induce $M D-2.2$ in vitro (22). This suggests that tissue inflammation may increase $M D-2.2$ expression that would negatively regulate LPS/TLR4 signaling. Further studies are needed to clarify this hypothesis.

We could not detect LBP mRNA in both primary gingival keratinocytes and fibroblasts cultured from healthy gingival tissues (Fig. 5). Also, E. coli LPS and/or hypoxia treatment used 
as simulators to mimic periodontal infection did not induce LBP transcripts in these cells (data not shown). The present results are in contrast to a previous immunohistochemistry study from our research group that LBP appeared to be expressed in the cytoplasm of gingival epithelial cells (23). However, LBP mRNA was detectable in healthy gingival tissue (Fig. 4f). It is likely that LBP peptide found in gingival tissue was principally produced in liver and then locally delivered via blood vessels, while the remaining LBP might be locally produced by gingival tissue cell types other than keratinocytes or fibroblasts.

It should be noted that primary cells used in this study were all cultured from healthy gingival tissues; theoretically, their behaviors might be different compared to those from periodontitis tissue. Observation from current report that in vivo TLR4 expression on junctional/pocket epithelia in periodontal health and disease (Fig. 3) remain not significant different despite TLR4 expression in pocket epithelium under inflammatory conditions tended to be higher. Further experiments with bigger sample size are needed to confirm such postulation.

\section{Conclusions}

This study provides strong evidence for the expression of TLR4, CD14 and MD-2 in both human gingival keratinocytes and fibroblasts. For the first time, we reported the expression levels of TLR4 protein in different periodontal epithelial compartments in vivo, in particular the lower expression level of the PAMP of interest in junctional epithelium indicating the 
possibility of heterogeneous nature of LPS-sensing in the dentogingival front in health.

\section{Acknowledgements}

This project was supported by the University of Hong Kong Small Project Funding (200807176129, 201007176307) and Seed Funding (200911159126). The authors report no conflict of interest related to this study. We would like to thanks Mr. C. Y. Yip and Dr. Edith Tong for their technical support and Mr. K. W. Shadow Yeung regarding assistant in statistical analysis. 


\section{References}

1. Jerala R. Structural biology of the LPS recognition. Int $J$ Med Microbiol 2007;297:353-363.

2. Kikkert R, Laine ML, Aarden LA, van Winkelhoff AJ. Activation of toll-like receptors 2 and 4 by gram-negative periodontal bacteria. Oral Microbiol Immunol 2007;22:145-151

3. Sugawara Y, Uehara A, Fujimoto Y, et al. Toll-like receptors, NOD1, and NOD2 in oral epithelial cells. J Dent Res 2006;85:524-529.

4. Uehara A, Sugawara S, Tamai R, Takada H. Contrasting responses of human gingival and colonic epithelial cells to lipopolysaccharides, lipoteichoic acids and peptidoglycans in the presence of soluble CD14. Med Microbiol Immunol 2001;189:185-192.

5. Uehara A, Sugawara Y, Kurata S, et al. Chemically synthesized pathogen-associated molecular patterns increase the expression of peptidoglycan recognition proteins via toll-like receptors, NOD1 and NOD2 in human oral epithelial cells. Cell Microbiol $2005 ; 7: 675-686$.

6. Uehara A, Fujimoto Y, Fukase K, Takada H. Various human epithelial cells express functional Toll-like receptors, NOD1 and NOD2 to produce anti-microbial peptides, but not proinflammatory cytokines. Mol Immunol 2007;44:3100-3111.

7. Sun Y, Guo QM, Liu DL, Zhang MZ, Shu R. In vivo expression of Toll-like receptor 2, Toll-like receptor 4, CSF2 and LY64 in Chinese chronic periodontitis patients. Oral Dis 
2010;16:343-350

8. Ren L, Leung WK, Darveau RP, Jin L. The expression profile of lipopolysaccharide-binding protein, membrane-bound CD14, and toll-like receptors 2 and 4 in chronic periodontitis. $J$ Periodontol 2005;76:1950-1959.

9. Beklen A, Hukkanen M, Richardson R, Konttinen YT. Immunohistochemical localization of Toll-like receptors 1-10 in periodontitis. Oral Microbiol Immunol 2008;23:425-431.

10. Mori Y, Yoshimura A, Ukai T, Lien E, Espevik T, Hara Y. Immunohistochemical localization of Toll-like receptors 2 and 4 in gingival tissue from patients with periodontitis. Oral Microbiol Immunol 2003;18:54-58.

11. Jaresova I, Rozkova D, Spisek R, Janda A, Brazova J, Sediva A. Kinetics of Toll-like receptor-4 splice variants expression in lipopolysaccharide-stimulated antigen presenting cells of healthy donors and patients with cystic fibrosis. Microbes Infect 2007;9:1359-1367.

12. Shimazu R, Akashi S, Ogata H, et al. MD-2, a molecule that confers lipopolysaccharide responsiveness on Toll-like receptor 4. J Exp Med 1999;189:1777-1782.

13. Gray P, Michelsen KS, Sirois CM, et al. Identification of a novel human MD-2 splice variant that negatively regulates Lipopolysaccharide-induced TLR4 signaling. $J$ Immunol 2010;184:6359-6366.

14. Ng KT, Li J-P, Ng KM, Tipoe GL, Leung WK, Fung M-L. Expression of hypoxia-inducible factor-1alpha in human periodontal tissue. $J$ Periodontol 
$2011 ; 82: 136-141$.

15. Li J-P, Li FYL, Xu A, et al. Lipopolysaccharide and hypoxia induced HIF-1 activation in human gingival fibroblasts. $J$ Periodontol 2012;83:816-824.

16. GenBank. Homo sapiens toll-like receptor 4 (TLR4), transcript variant 2, mRNA. http://www.ncbi.nlm.nih.gov/nuccore/NM_138556.1?report=genbank, accessed December 2012.

17. Iwami KI, Matsuguchi T, Masuda A, Kikuchi T, Musikacharoen T, Yoshikai Y. Cutting edge: naturally occurring soluble form of mouse Toll-like receptor 4 inhibits lipopolysaccharide signaling. J Immunol 2000; 165: 6682-6686.

18. da Silva Correia J, Ulevitch RJ. MD-2 and TLR4 N-linked glycosylations are important for a functional lipopolysaccharide receptor. J Biol Chem 2002;277:1845-1854.

19. da Silva Correia J, Soldau K, Christen U, Tobias PS, Ulevitch RJ. Lipopolysaccharide is in close proximity to each of the proteins in its membrane receptor complex. transfer from CD14 to TLR4 and MD-2. J Biol Chem 2001;276:21129-21135.

20. Muroi M, Ohnishi T, Tanamoto K. Regions of the mouse CD14 molecule required for toll-like receptor 2- and 4-mediated activation of NF-kappa B. J Biol Chem 2002;277:42372-42379.

21. Xia Y, Yamagata K, Krukoff TL. Differential expression of the CD14/TLR4 complex and inflammatory signaling molecules following i.c.v. administration of LPS. Brain Res 2006;1095:85-95.

22. Gray P, Michelsen KS, Sirois CM, et al. Identification of a novel human MD-2 splice 
1

2

3

4

5

6

7

8

9

10

11

12

13

14

15

16

17

18

19

20

21

22

23

24

25

26

27

28

29

30

31

32

33

34

35

36

37

38

39

40

41

42

43

44

45

46

47

48

49

50

51

52

53

54

55

56

57

58

59

60 variant that negatively regulates Lipopolysaccharide-induced TLR4 signaling. $J$ Immunol 2010;184:6359-6366.

23. Ren L, Jin L, Leung WK. Local expression of lipopolysaccharide-binding protein in human gingival tissues. J Periodontal Res 2004;39:242-248. 


\section{Legends}

Fig. 1. TLR4 protein expression profiles in healthy gingiva (DAB stain. Scale bars represent $200 \mu \mathrm{m}, 50 \mu \mathrm{m}$ or $25 \mu \mathrm{m}$ as indicated). a) micrograph of healthy gingiva. b) to e) show magnified regions. TLR4-immunoreactivity could be found in oral epithelium (OE; b) and oral sulcular epithelium (OSE; b, c \& e), but occasional in junctional epithelium (JE; d). Fibroblast-like cells (open arrows; c, d) show slight TLR4-immunoreactivity. Leukocyte-like cells (open and solid arrow heads) were scattered in OSE, JE and subepithelial regions of healthy gingiva (c, d). Most leukocyte-like cells including those epithelium-infiltrating were not TLR4-immunoreactive (open arrow heads), except those with dendritic morphology were TLR4 positive (solid arrow heads). The TLR4-positive staining in endothelial cells (arrows) was very weak or absent.

Fig. 2. TLR4 protein expression profiles in shallow (A) or deep (B) periodontal pockets (DAB stain. Scale bars represent $200 \mu \mathrm{m}, 50 \mu \mathrm{m}$ or $25 \mu \mathrm{m}$ as indicated). a) is a section of shallow periodontal pocket biopsy. b) to d) show magnified regions. e) is a section of deep periodontal pocket biopsy f) to j) show magnified regions. TLR4-immunoreactivity could be found in oral epithelium (OE; d) and pocket epithelium (PE; c, g). Compared to healthy gingiva (Fig. 1c, 1d) where fibroblasts were mostly free of TLR4-immunoreactivity, fibroblast-like cells in periodontitis tissue (f, i; open arrows) proximal to inflammatory front were heavily stained. Leukocyte-like cells (open and solid arrow head) appeared densely 
infiltrated the periodontitis tissue $(f, j)$. Although most epithelium-infiltrating leukocyte-like cells were not TLR4-immunoreactive (open arrow head), those in the subepithelial region were more intensively stained (solid arrow head) than in healthy gingiva (Fig. 1d). TLR4-positive inflammatory cells appeared to accumulate around the front of the periodontitis lesion $(\mathrm{h}, \mathrm{k})$, which are characterized by a mass of densely infiltrating leukocytes. The proportion of subepithelial cells that were TLR4-positive was significantly greater in periodontitis tissue than that in healthy controls (compared to Fig. 1: $68.4 \pm 13.7 \%$ in 38 periodontal pockets vs. $12.3 \pm 5.6 \%$ in 10 control samples; $P<0.001, t$-test. ).

Fig. 3. Area TLR4 positive (mean \pm SD percentage proportion) at various epithelial compartments observable in histological sections of periodontal biopsies. Significant higher proportions of area positively stained for TLR4 [area TLR4 positive (\%)] were observable at healthy oral epithelium $(\mathrm{H}-\mathrm{OE}, \mathrm{n}=10)$, oral sulcular epithelium (H-OSE, $\mathrm{n}=10$ ) and periodontitis oral epithelium (P-OE, n=38), compared to junctional epithelium (H-JE, n=10; one-way ANOVA with Bonferroni multiple comparisons, $* * P=0.01, * * * P=0.001$ ). Area TLR4 positive $(\%)$ in pocket epithelium (P-PE, $n=38)$ was not statistically different to that of other diseased or healthy epithelial compartments.

Fig. 4. The mRNA expression of TLR4, MD-2, CD14 and LBP in primary human gingival keratinocytes (HGK), fibroblasts (HGF) and healthy gingival tissue (HGT). a) Human TLR4 gene includes two exons $a$ and $b$, which results in four possible splicing variants. b) Possible 
splicing variants TLR4.1, TLR4.2, TLR4.3 and TLR4.4. A pair of PCR primers (P1 and P2) was designed to amplify the splicing variants containing exons $a$ and $b$. c) Three TLR4 splicing variants, TLR4.3, TLR4.1 and TLR4.4 were found in HGF, HGK and HGT. d) Two mRNA splicing variants of MD-2, MD-2.1 (containing the splicing exon) and $M D-2.2$ (lacking the splicing exon) were found in HGF, HGK and HGT. MD-2.1 appears to be expressed more in gingival cells and tissue. e) CD14 mRNA was detected in HGF, HGK and HGT. f) LBP mRNA was found in HGT, but not in HGK or HGF.

Fig. 5. Protein expression of CD14, MD-2 and TLR4 in human gingival tissue and cells (A) and association between CD14, MD-2 and TLR4 peptides expressed in HGF and HGK (B). Proteins were immunoprecipitated (IP) and immunoblotted (IB) from the whole cell or tissue lysate $(500 \mu \mathrm{g}$ cellular protein or $800 \mu \mathrm{g}$ tissue protein for TLR4 and CD14, $20 \mu \mathrm{g}$ protein for MD-2). The anti-human antibody species were Rb: rabbit, Ms: mouse and Gt: goat. BSA: bovine serum albumin $(500 \mu \mathrm{g})$ negative control lane. A. Protein expression of a) CD14, b) MD-2 and c) TLR4 in human peripheral blood mononuclear cells (PBMC, positive control), human gingival fibroblasts (HGF), human gingival keratinocytes (HGK), healthy gingival tissue (HGT) and periodontitis gingival tissue (PGT). B. The association between CD14, MD-2 and TLR4 proteins expressed in HGF and HGK. Whole HGF, HGK and PBMC (positive control) lysate (500 $\mu \mathrm{g}$ protein each) and BSA (500 $\mu \mathrm{g}$, negative control) were IP with anti-CD14 (e), -MD-2 (g) or -TLR4 (d, f) antibody, and IB with the relevant anti-human antibody. d) and e) TLR4 and CD14 peptides were co-immunoprecipitated. f) and g) MD-2 
and TLR4 peptides were co-immunoprecipitated. CD14 and MD-2 did not co-immunoprecipitate (data not shown), indicating the two molecules are not directly interacting with each other.

Supplementary Fig. S1. Semi-quantification of positive immunohistochemical (IHC) staining. Panel A. Field selections in anti-TLR4 antibodies stained healthy gingival tissue: a (corresponding to Fig. 1b), b (corresponding to Fig. 1d) or periodontitis tissue: c (corresponding to Fig. 2b), d (corresponding to Fig. 2g). Non-overlapping fields of interest (FOI) [oral epithelium (OE)/oral sulcular epithelium (OSE): 5,000-30,000 $\mu^{2}$, junctional epithelium (JE)/pocket epithelium (PE): 5,000-15,000 $\mu \mathrm{m}^{2}$, or deep PE: 5,000-75,000 $\mu \mathrm{m}^{2}$ rectangular boxes) were defined in antibodies stained image sections: JE (g), OE (f, i), OSE (e) and PE (h, j). The TLR4 IHC staining quantification was as described in Panel B. k: Defining a FOI via drawing a rectangle in a digital image acquired from a stained section (shown is an OSE section); 1: Assigning a surrogate color (in this case blue color was selected) for DAB stain (brown) indicating positive immunolabeling; m: Using Leica Qwin Standard V 2.6 software (Leica, Wetzlar, Germany) to measure the area percentage (Area\%). 

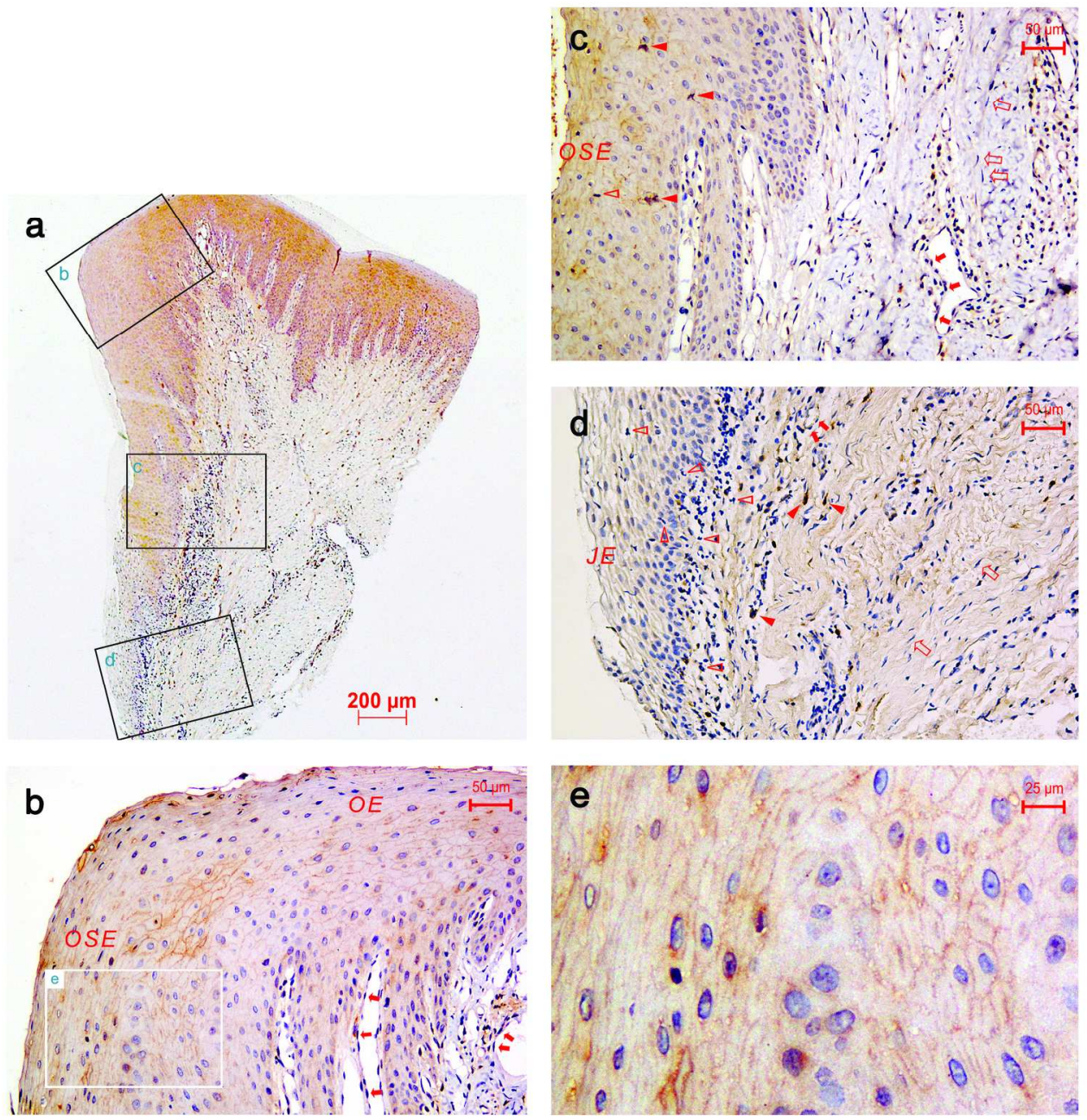

$185 \times 192 \mathrm{~mm}(300 \times 300 \mathrm{DPI})$

Journal of Periodontal Research 


1
2
3
4
5
6
7
8
9
10
11
12
13
14
15
16
17
18
19
20
21
22
23
24
25
26
27
28
29
30
31
32
33
34
35
36
37
38
39
40
41
42
43
44
45
46
47
48
49
50
51
52
53
54
55
56
57
58
59
60

A a

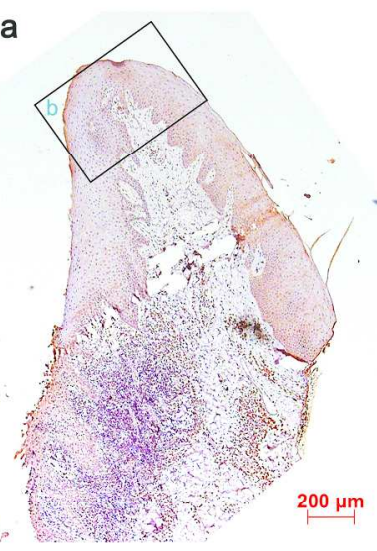

b

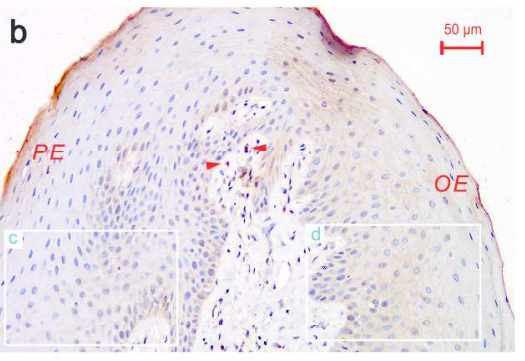

c , log a d

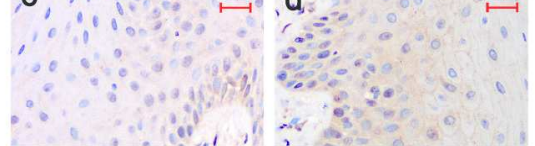

B e
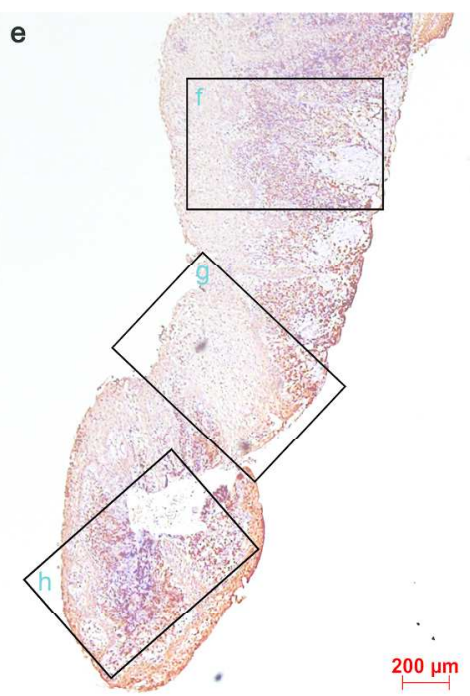

$200 \mu \mathrm{m}$

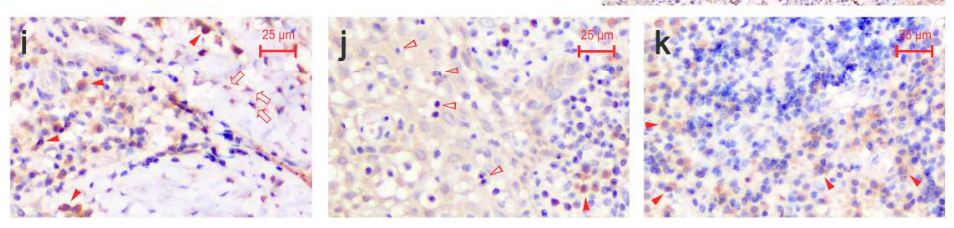

$181 \times 273 \mathrm{~mm}(300 \times 300 \mathrm{DPI})$

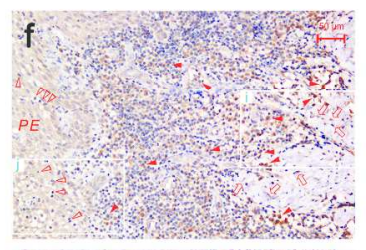

g

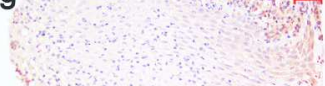
and 9
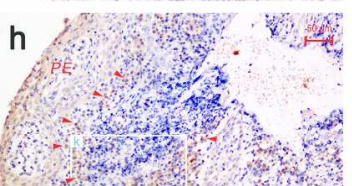

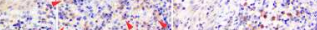

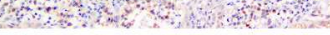

47

48

49

51

52

53

54

55

56

57 


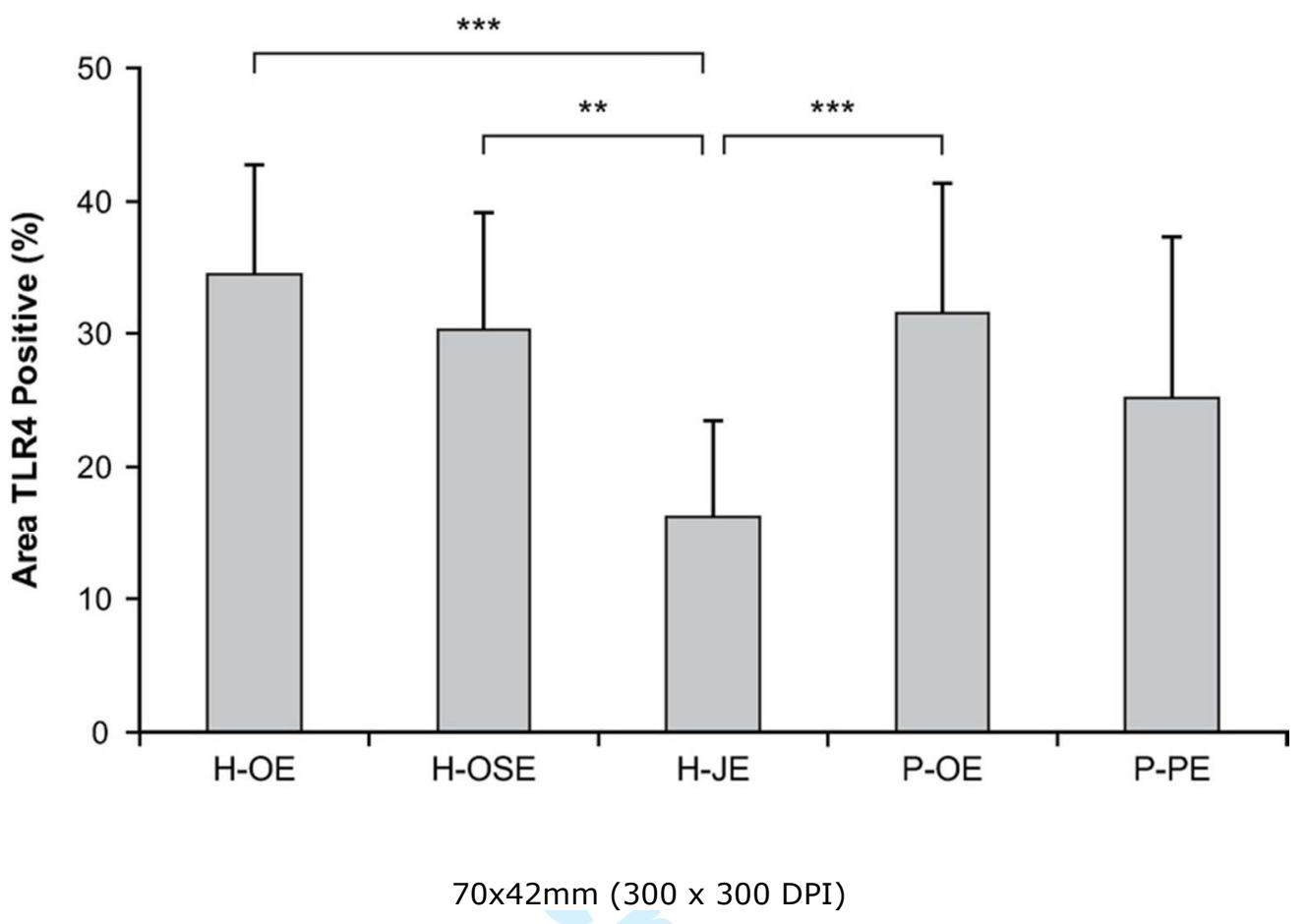

29

35

36

37

38

39

40

41

42

43

44

45

46

47

48

49

50

51

52

53

54

55

56

57

58

59

60

Journal of Periodontal Research 
a

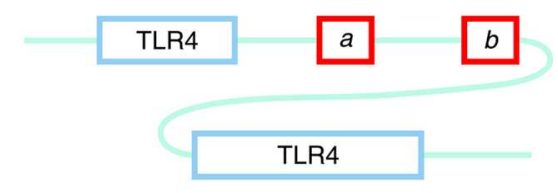

b

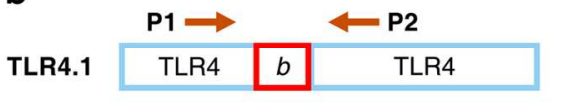

\begin{tabular}{l|l|l|l|}
\hline TLR4.2 & TLR4 & $a$ & TLR4 \\
\hline
\end{tabular}

\begin{tabular}{|l|l|l|l|l|}
\hline TLR4.3 & TLR4 & $a$ & $b$ & TLR4 \\
\hline
\end{tabular}

\begin{tabular}{|l|l|l|}
\hline TLR4.4 & TLR4 & TLR4 \\
\hline
\end{tabular}

C

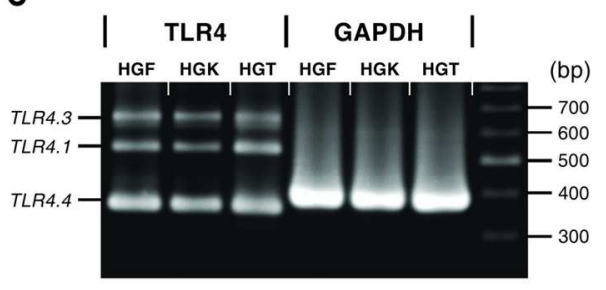

d

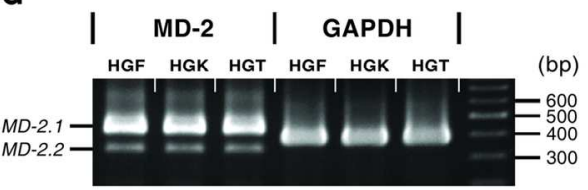

e

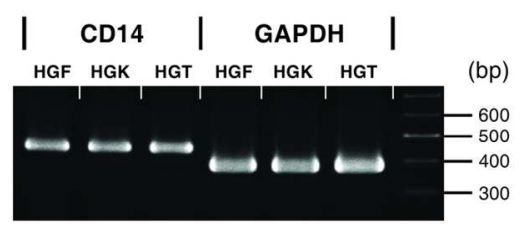

f

\begin{tabular}{ll|c|} 
LBP & GAPDH \\
HGF HGK HGT HGF HGK HGT
\end{tabular}

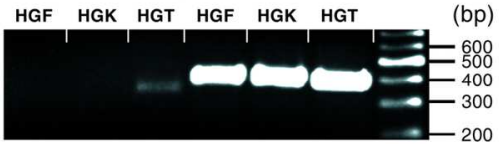

$146 \times 118 \mathrm{~mm}(300 \times 300$ DPI $)$ 
A

B

a

IB: CD14 Ms

PBMC HGF HGK HGT PGT BSA (kDa)

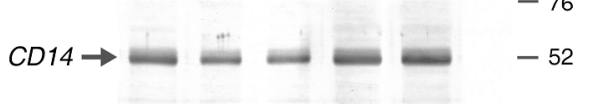

$-38$

16

17

18

19

20

21

22

23

24

25

26

27

28

29

30

31

32

33

34

35

36

37

38

39

40

41

42

43

44

45

46

47

48

49

50

51

52

53

54

55

56

57

58

59

60

b

IB: $M D-2 R b$

PBMC HGF HGK HGT PGT BSA (kDa)

$-31$
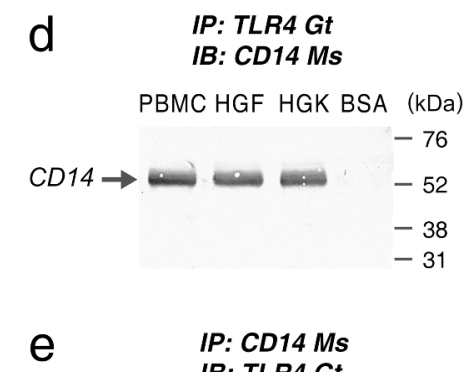

IB: TLR4 Gt

PBMC HGF HGK BSA (kDa)

$-150$

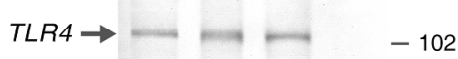

$-76$

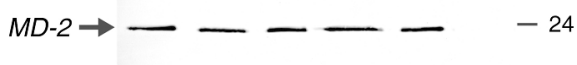

$-17$

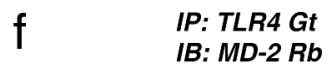

PBMC HGF HGK BSA (kDa)

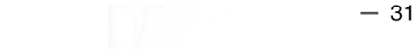

$M D-2 \rightarrow--\longrightarrow-24$

$-17$

C

IP: TLR4 Gt

IB: TLR4 Ms

$9 \quad I P: M D-2 R b$

IB: TLR4 Gt

PBMC HGF HGK HGT PGT BSA (kDa)

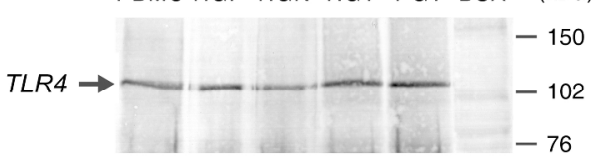

PBMCHGF HGK BSA (kDa)

$-150$

$T L R 4 \rightarrow--=-102$

$-76$

Figure 5

$133 \times 144 \mathrm{~mm}(600 \times 600 \mathrm{DPI})$

Journal of Periodontal Research 
A a
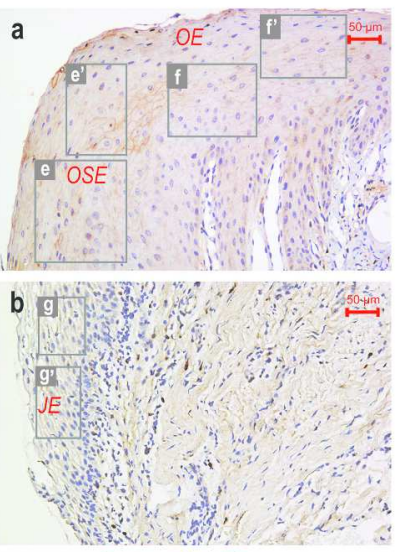

c
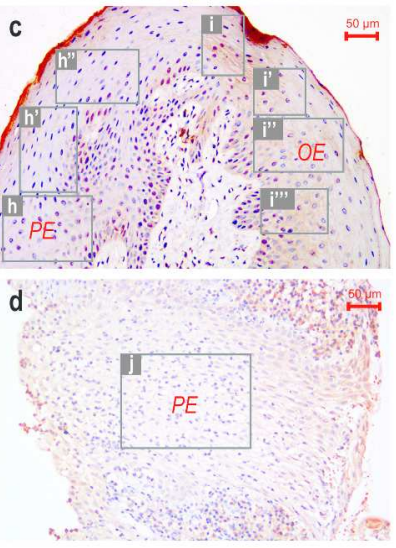

B $k$

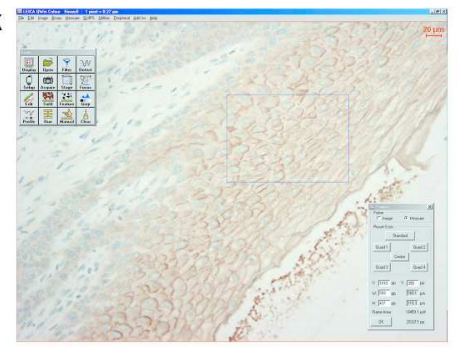

I

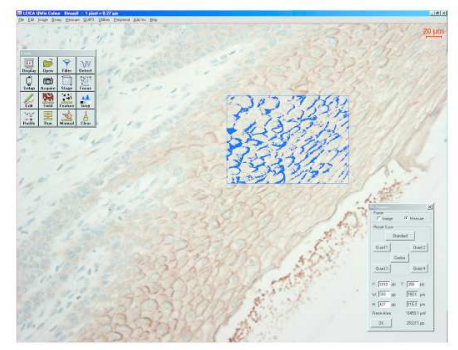

m

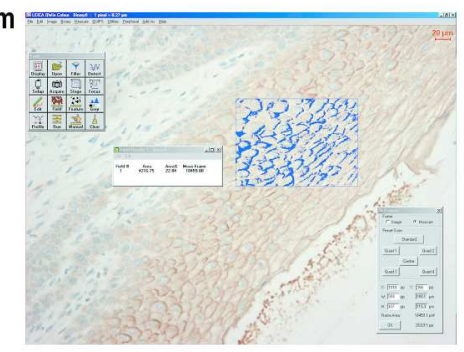

Supplementary Fig. S1. Semi-quantification of positive immunohistochemical (IHC) staining. Panel A. Ficld selections in anti-TLR4 antibodies stained healthy gingival tissue: a (corresponding to Fig. $\mathrm{lb}$ ), b (corresponding to Fig. 1d) or pcriodontitis tissuc: $c$ (corrcsponding to Fig. 2b), d (corrcsponding to Fig. 2g). Non-overlapping ficlds of interest (FOI) [oral epithelium (OE)/oral sulcular epithelium (OSE): $5,000-30,000 \mu \mathrm{m}^{2}$, junctional epithelium (JE)/pocket epithelium (PE): 5,000-15,000 $\mathrm{m}^{2}$, or deep PE: $5,000-75,000 \mu^{2}$ rectangular boxes) were defined in antibodies stained image sections: JE (g), OE (f, i), OSE (e) and PE (h, j). The TLR4 IHC staining quantification was as described in Panel B. k: Defining a FOI via drawing a rectangle in a digital image acquired from a stained section (shown is an OSE section); l: Assigning a surrogate color (in this case blue color was selected) for DAB stain (brown) indicating positive immunolabeling; m: Using Leica Qwin Standard V 2.6 software (Leica, Wetzlar, Germany) to measure the area percentage (Area\%)

209×297mm (300 x 300 DPI) 